DOI: 10.20472/IAC.2018.036.016

TRACY HEAVNER

University of South Alabama, United States

\title{
JAZZ PEDAGOGY: INCORPORATING AUTHENTIC JAZZ ARTICULATIONS INTO SOLO AND JAZZ ENSEMBLE PERFORMANCE / FLUTE PERFORMANCE
}

\begin{abstract}
:
When performing jazz music, especially jazz in the swing style, utilizing authentic articulation is essential. However, in many jazz arrangements, specific articulations are not notated in the ensemble parts. In addition, when performing improvised solos, ensemble members must create their own articulations based upon their knowledge of articulation style, the tempo of the tune and the rhythms they choose to play. Since many students are not knowledgeable in this area, they must rely on their jazz ensemble director to supply them with the information necessary to perform with correct articulation. Unfortunately, many jazz ensemble directors also do not have the knowledge necessary to instruct students on how to properly articulate in the jazz style. As a result, students do not use authentic articulations when performing their jazz ensemble parts or when improvising solos. The purpose of this paper is to provide jazz ensemble directors and ensemble members with the information necessary to incorporate correct, authentic jazz swing articulations into solo and ensemble performance. There are five basic types of jazz articulations used when playing jazz swing music. They are back-accent, hard, slur, half and doodle tonguing. These articulations can be used separately but are most commonly played in combination with each other to provide the most authentic jazz articulation style. Using correct jazz articulations will allow students to perform in a more authentic jazz style, greatly enhancing their performance skills and their understanding of the jazz idiom.
\end{abstract}

\section{Keywords:}

Jazz Pedagogy, Teacher Education

JEL Classification: 129 


\section{Introduction}

When performing jazz music, especially jazz in the swing style, utilizing authentic articulation is essential. The use of proper articulation is very important because it assists the musical lines with clarity, improves the swing feel and provides intensity and interest to the performance. When comparing professional jazz artists to that of advancing performers, one of the most dramatic differences between the two, in addition to note and rhythm selection, is articulation.

\section{Statement of the Problem}

In many jazz arrangements, specific articulations are not notated in the ensemble parts. In addition, when performing improvised solos, ensemble members must create their own articulations based upon their knowledge of articulation style, the tempo of the tune and the rhythms they choose to play. Since many students are not knowledgeable in this area, they rely on their jazz ensemble director to supply them with the information necessary to perform with the correct articulations. Unfortunately, many jazz ensemble directors also do not have the knowledge necessary to properly instruct their students on how to properly articulate in the jazz style. As a result, students do not use authentic articulations when performing their jazz ensemble parts or when improvising solos.

\section{Purpose of Study}

The purpose of this study is to provide jazz ensemble directors with the information necessary to incorporate correct, authentic jazz swing articulations into solo and ensemble performance. Using correct jazz articulation will allow students to perform in a more authentic jazz style, greatly enhancing their performance skills and their understanding of the jazz idiom.

\section{What is Swinging?}

Some musicians easily grasp the concept of swinging while others seem to struggle. In reality, swinging is not a difficult concept to understand if explained correctly. However, applying this concept to performance may be more difficult. When reading pairs of eighth notes in classical music, the beat is divided equally between the two. When reading music in a swing style, the performer will see pairs of eighth notes written in the same manner as in classical music. However, somewhere on the written page will be directions for the performer to swing these notes. This requires the musician to interpret the notation actually playing rhythms that are not written on the page. When playing a pair of eighth notes in a swing style, the performer is actually performing the rhythm of three triplet eighth notes with the first two tied together. The tying of the first two triplet eighth notes 
allows the rhythm to swing by dividing the beat into two unequal divisions. The first eighth note is worth two thirds of the beat while the last eighth note is worth one third of the beat.

Tied Eighth/Eighth Triplet $=$ Quarter/Eighth Triplet $=$ Swing Eighth Notes
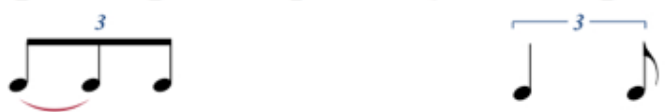

\section{Learning to Swing}

When initially practicing swing eighth notes, the performer should first count and then slowly play three triplet eighth notes to establish an even triplet feel.

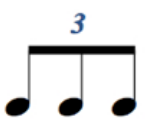

Tri-pa- let

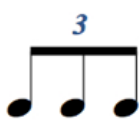

Tri- pa- let

When this rhythm feels comfortable, then count and play the actual swing eighth note pattern of tri-plet, tri-plet with the "tri" getting two thirds of the beat while the "plet" gets one third.

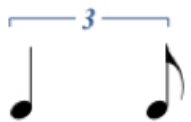

Tri- plet

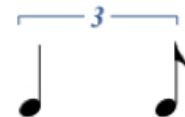

Tri- plet

Once this basic swing feel has been developed, the performer should practice performing a variety of scales, patterns, exercises and etudes in the swing style at a variety of tempi.

\section{Swinging at Different Tempi}

Perhaps the most difficult aspect of swinging and the problem that troubles many performers is the ability to swing at a variety of tempi. When performing at a slow tempo, students will play eighth notes using the triplet division discussed earlier. However, as the tempo becomes faster, the eighth notes must be played more evenly to retain a legato, flowing jazz style. At very fast tempi, swing eighth notes do not actually swing at all, but are divided and played evenly. This concept of playing the eighth notes more evenly as the tempo increases causes problems for multitudes of performers. Many times, students will try to retain the triplet division used at a slow tempo for faster tunes producing an 
awkward, unnatural, overly swung style. Other times performers will play eighth notes too evenly at a slow tempo causing the tune not to swing enough.

The ability to know how much to swing eighth notes at a variety of tempi and to get the musical line to swing hard and sound natural is one of the most difficult tasks jazz musicians have to perform. To develop this ability, students should first listen to recordings of master jazz musicians improvising at a variety of tempi paying close attention to how the eighth notes are played and what changes are made from one tempo to another. To assist in this process, the performer should either transcribe or attain a transcription of the solos being listened to. Next students should imitate these musicians trying to perform the musical lines in exactly same manner including dynamics and articulations. Finally, the performer should play along with the recordings to get a feel for how the lines are phrased. If possible, performers should record themselves performing these solos and compare their performance to the original. By practicing this way, the ability to swing at a variety of tempi will be developed and become a natural part of a musician's playing style.

\section{Types of Jazz Articulations}

There are five basic types of jazz articulations used when playing jazz swing music. They are back-accent, hard, slurring, half tonguing and doodle tonguing. These articulations can be used separately but are most commonly played in combination with each other to provide the most authentic jazz articulation style. The most common mistakes performers make when incorrectly articulating jazz swing music are tonguing every note when performing tunes at slower tempi, slurring every note at faster tempi and randomly tonguing or slurring notes when improvising solos. These common errors impede a player's musical performance and also result in a performance that is not a authentic representation of the jazz swing idiom.

\section{Back-Accent Articulation}

When playing jazz in the swing style, back-accent tonguing is the most commonly used articulation when playing swing music. Back-accent tonguing is very easy to understand and will quickly improve the performer's ability to perform clean lines and authentic sounding musical phrases. When using this articulation, the musician lightly tongues each note on the upbeat and slurs to the note on the beat. For example, in a series of eighth notes starting on the upbeat, the performer should tongue the first eighth note in the series and then slur to the note on the beat. This pattern should be repeated until the series of eighth notes ends. 


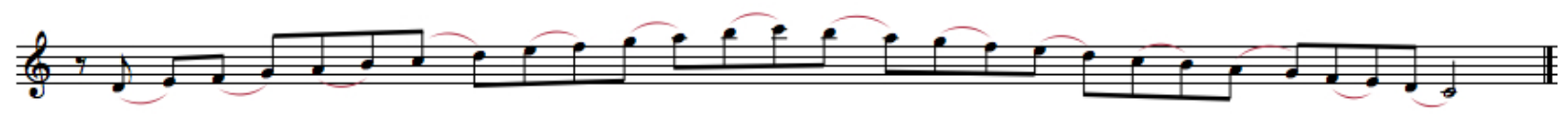

If a series of eighth notes begins on the beat, the performer should tongue the first two eighth notes in the series, the first eighth note on the beat to start the series and also the second eighth note, since it is on the upbeat, and then slur to the next note on the beat.

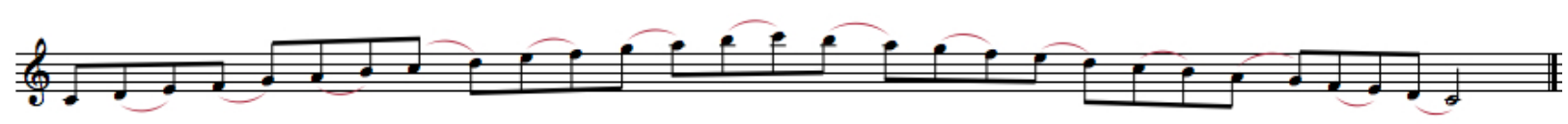

Back-accent tonguing assists the musical phrase in swinging because all upbeat notes are being lightly tongued while all notes on the beats are being slurred. It should be emphasized that when using this style of articulation, the musician should lightly tongue each upbeat eighth note and play in a legato style. Notes should be sustained at all times producing a continuous flow of sound. When players incorrectly perform back-accent tonguing, the two most common problems are tonguing to hard and allowing the tone to stop between the slurred note on the beat and the tongued note on the following upbeat.

Since the concept of back-accent tonguing is easy to understand and produces authentically articulated phrases, it is also a great tool to use when performing in sectional playing of a jazz ensemble. If each member of the section understands and correctly uses back-accent tonguing, the section will have a consistent articulation that promotes hard swinging lines but with a minimum amount of practice.

To practice back-accent tonguing, performers should first practice a variety of scales and modes used for jazz improvisation to get accustomed to using this style of articulation. Then this articulation can be incorporated into playing ensemble parts and improvisation. After back-accent tonguing has been mastered, players may want to vary their articulation, customizing it based upon the notes and specific rhythms being played and also through imitation of articulations performed by prominent jazz players.

\section{Hard Articulation}

Hard articulation consists of tonguing all notes in a particular passage. This is a common articulation pattern in many styles of music but is used somewhat sparingly in playing jazz swing, especially when performing music at faster tempi. However, many performers use combine this type of articulation combined with back-accent, slurring and half tonguing to produce an authentic articulation style. 


\section{Slur Articulation}

Slurring is a style of articulation in which only the first note in a group of notes is tongued. The rest of the notes are played in a connected, legato style in which there are no breaks in the air stream. Since jazz is played in a legato style, this articulation works well however, slurring to many notes in a row will not give the jazz phrase enough clarity. Therefore, most jazz performers combine the slurring articulation with other types of articulations to provide clarity and more variety to the melodic line.

\section{Half Tonquing}

Half tonguing is a style of articulation used in the performance of jazz that adds much expression to a musical phrase and also promotes a swing feel. It is used by many jazz masters as well as prominent professional musicians and will greatly enhance a performer's playing style. Half tonguing is basically a tool for muting specific notes in a jazz phrase using the tongue instead of manipulating the air stream. Muting specific notes at key points in a musical phrase allows the phrase to swing harder and also brings more attention to the melodic notes enhancing the melodic line. Also, this method of muting notes is much more efficient than using the air stream since when performing fast, articulated lines, it is very difficult to manipulate single notes by adjusting the air flow.

To develop the ability to half tongue, performers should first think and say the syllables Doo-N, Doo-N. This will simulate the tongue movement that occurs when actually performing the half-tongued articulation. Next, the performer should attempt to half tongue a note in the middle register of the instrument by initially playing and holding the selected note. be pulled away and the process started over.

After half tonguing has been mastered on notes in the middle register, the performer can expand this articulation to notes in the upper and lower registers. The next step is to half tongue notes of shorter duration and to develop coordination between the tongue and the fingers. One exercise for doing this is to slowly play a $G$ major scale in eighth notes, tonguing the notes on the beat and half tonguing the notes on the upbeats. After this pattern becomes natural, it can be performed using other major scales.

A jazz articulation pattern that is commonly used in running eighth note lines combines half tonguing with back-accent tonguing to produce a clean, hard swinging phrase. In this pattern, back-accent tonguing is used to start a series of eighth notes but on the fourth note of the series, the note is half tongued instead of being slurred. When performing this articulation pattern the performer should think of the syllables Doo-Doo-Oo-N. 


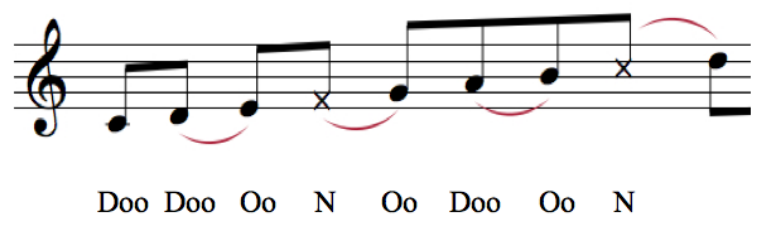

Another common jazz articulation combines half tonguing with back-accent tonguing using the same basic pattern previously discussed but in this articulation, the second note in the series is half tongued instead of the fourth. Syllables for this pattern are Doo-N-OoDoo.

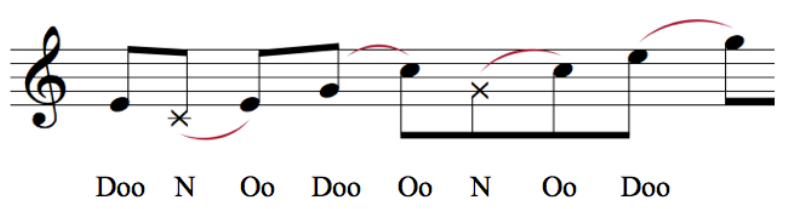

The process of half tonguing may feel strange in the beginning but after a short period of time, the tongue will become accustomed to this technique and it will become a commonly used articulation pattern.

\section{Doodle Tonguing}

Doodle tonguing is a jazz articulation used by brass players that allows them to play fast, legato, articulated musical lines with little effort. This technique is called Doodle Tonguing because when properly executed, the performer's tongue moves as if saying doo-dle, doo-dle, doo-dle, or do-ul, do-ul, do-ul. When mastered, jazz performers will be able to legato articulate every note in a musical line at an extremely fast tempo, allowing them to have great facility on their instrument.

\section{Summary}

To properly perform jazz swing music, utilizing authentic articulation is very important to the jazz swing style. Unfortunately, many jazz ensemble members do not have the knowledge and skill necessary to properly perform this music with correct articulations. In addition, their jazz ensemble directors, in which these ensemble members rely on for proper instruction, also lack the knowledge and skill to teach their ensemble members on how to properly articulate this style of music. It is hoped that the information presented in this paper will assist jazz ensemble directors and their students to incorporate authentic combinations of the five basic articulations, back-accent, hard, slurring, half tonguing and doodle tonguing, into their solo and ensemble performance allowing them to perform jazz swing music at the highest level. 


\section{References}

Barrick, C. (2016). Can you Dig? Teaching Jazz Articulation and Style. https://nafme.org/teaching-iazzarticulation-style/

Clark, C. (2016). Teaching Jazz Articulation to Young Brass Players.

https://www.smartmusic.com/blog/teaching-jazz-articulation-to-young-brass-players/

Heavner, T. (2013). Saxophone Secrets: 60 Performance Strategies for the Advanced Saxophonist. Scarecrow Music Press, Lanham, Maryland.

Lebrun, M. (2014). Tonguing for Jazz Saxophone. http://thewoodshedmusic.com/2014/04/tonguing-for-jazzsaxophone/

Tolsen, J. (2012). Jazz Style and Articulation: How to Get Your Band or Choir to Swing.

http://mej.sagepub.com/content/99/1/80. 